\title{
Experimental Evidence for Two- Dimensional Ostwald Ripening in Semiconductor Nanoplatelets
}

\section{Journal Article}

\section{Author(s):}

Knüsel, Philippe N.; Riedinger, Andreas; Rossinelli, Aurelio (D); Ott, Florian D.; Mule, Aniket S.; Norris, David J.

Publication date:

2020

Permanent link:

https://doi.org/10.3929/ethz-b-000408529

Rights / license:

In Copyright - Non-Commercial Use Permitted

Originally published in:

Chemistry of Materials 32(7), https://doi.org/10.1021/acs.chemmater.0c01238

\section{Funding acknowledgement:}

165559 - Optical Strong Coupling in Colloidal Quantum Dots (SNF)

339905 - Quantum-Dot Plasmonics and Spasers (EC) 


\title{
Experimental Evidence for Two-Dimensional Ostwald Ripening in Semiconductor Nanoplatelets
}

\author{
Philippe N. Knüsel, ${ }^{\dagger}$ Andreas Riedinger,,$^{\dagger}$, Aurelio A. Rossinelli, ${ }^{\dagger}$ Florian D. Ott, ${ }^{\dagger}$ Aniket S. Mule, ${ }^{\dagger}$ \\ and David J. Norris ${ }^{*, \dagger}$ \\ ${ }^{\dagger}$ Optical Materials Engineering Laboratory, Department of Mechanical and Process Engineering, ETH Zurich, 8092 Zurich, \\ Switzerland \\ ${ }^{\ddagger}$ Max Planck Institute for Polymer Research, Ackermannweg 10, 55128 Mainz, Germany
}

\begin{abstract}
Colloidal semiconductor nanoplatelets are rectangular, quasi-two-dimensional crystallites that are only a few atomic layers thick. In the most heavily studied systems ( such as $\mathrm{CdE}$ with $\mathrm{E}=\mathrm{S}, \mathrm{Se}$, or $\mathrm{Te}$ ), the highly anisotropic nanoplatelet shape forms from an isotropic cubic crystal lattice. This has been difficult to reconcile with standard nanocrystal growth models. Previously, we proposed that nanoplatelets arise due to an intrinsic kinetic instability that enhances growth on narrow crystal facets under surface-reaction-limited conditions. Here, we test this model experimentally by synthesizing small "baby" CdS nanoplatelets and performing ripening experiments. During heating, we observe transitions to thicker nanoplatelet populations that are consistent with two-dimensional Ostwald ripening. We then heat CdSe nanoplatelets in the presence of "baby" CdS nanoplatelets to form CdSe-CdS core-crown nanoplatelets. This indicates that individual nanoplatelets dissolve and transfer their material to thicker nanoplatelets, consistent with the kinetic instability model. Finally, we grow thin films of CdS nanoplatelets by combining "baby" CdS nanoplatelets with cadmium carboxylate and heating on a substrate. This shows that Ostwald ripening may also be exploited as a facile and versatile approach to nanoplatelet growth.
\end{abstract}

\section{INTRODUCTION}

Methods to prepare nanometer-scale semiconductor crystallites via colloidal chemistry are now highly developed. ${ }^{1,2}$ In particular, nanocrystals that exhibit a quasi-spherical shape (typically referred to as quantum dots, $\mathrm{QDs}^{3}$ ) have been prepared from many materials. QDs have been investigated because their electronic transitions are strongly dependent on their diameter, ${ }^{46}$ providing useful sizetunable optical properties (e.g., absorption and fluorescence). However, if the QD size varies within a sample, spectral broadening occurs that is detrimental for many applications. Consequently, much work has aimed at reducing the QD size distribution during growth. ${ }^{1,2}$ This includes efforts to understand and reduce the negative effect of Ostwald ripening. ${ }^{7}$ Such ripening is caused by the size-dependent solubility of nanocrystals. Under conditions of depleted reactants, smaller particles in a sample can dissolve and shrink while larger particles remain stable and grow. Because this broadens (or "defocuses") the size distribution, synthetic protocols to avoid Ostwald ripening have been developed. ${ }^{8}$ Nevertheless, the size distribution in real $\mathrm{QD}$ samples is never fully eliminated. Even in the best cases, the diameter varies by $\sim 5 \%$.

Such residual size inhomogeneities in semiconductor nanocrystals have been assumed to be unavoidable. Indeed, as other nanocrystal shapes have been introduced over the years (nanorods, ${ }^{9}$ nanowires, ${ }^{10-12}$ tetrapods, ${ }^{13-15}$ cubes, ${ }^{16}$ etc.), each has suffered from distributions in size. However, a decade ago, an exception was discovered-semiconductor nanoplatelets (NPLs). ${ }^{17}$ These nanocrystals are rectangular plates with a uniform atomic-scale thickness. Moreover, samples can be prepared in which all the NPLs have the same thickness (e.g., 3 monolayers). Because their optical properties are governed by this thickness, they can exhibit better optical properties than QDs, including narrower fluorescence linewidths ${ }^{18}$ and larger absorption cross sections. ${ }^{19}$ Thus, NPLs have been studied not only to understand the optical ${ }^{20}$ and electronic ${ }^{21}$ properties of quasi-two-dimensional (quasi-2D) semiconductors, but also for applications in light-emitting devices ${ }^{22}$ and lasers. ${ }^{23}$

Since the first report of CdSe NPLs, ${ }^{17}$ subsequent research has led to the control of their lateral shape ${ }^{24}$ the development of new NPL materials (e.g., lead and zinc chalcogenides ${ }^{25}$ and mercury telluride ${ }^{26}$ ), and the growth of NPL heterostructures (e.g., corecrown $^{27}$ and core-shell ${ }^{28-30}$ ). During these developments, other quasi-2D semiconductor nanocrystals, including nanoribbons, ${ }^{31}$ nanosheets, ${ }^{32}$ and quantum belts ${ }^{33}$ have also been studied in parallel. However, during all of this work, the formation mechanism behind NPLs and their uniform thickness remained poorly understood. For CdSe NPLs exhibiting the zincblende (cubic) crystal structure, oriented attachment of preformed nanoclusters to a growing crystallite has been hypothesized, ${ }^{33}$ but experimental results suggest continuous lateral growth from CdSe "monomers. ${ }^{34-36}$ For this latter growth mode, it is then unclear how an isotropic crystal lattice generates such a highly anisotropic quasi-2D shape. Templated growth within soft molecular lamellae was proposed as a possible route to break symmetry, ${ }^{37}$ or selective binding of at least two different ligands to the surface facets of isotropic crystalline seeds. ${ }^{18}$ However, quantitative models based on these ideas have not yet appeared.

Another puzzle has been why the thickness distribution in NPL samples does not broaden during growth. Prolonged heating of reaction mixtures simply causes NPLs to increase slowly in thickness from $m$ to $m+1$ layers. ${ }^{38-40}$ (We use the common convention that an $m$-monolayer-thick CdE NPL contains $m+1$ layers of $\mathrm{Cd}$ and $m$ layers of $\mathrm{E}=\mathrm{S}$, Se, or Te.) Thus, the thickness of NPL samples can be well controlled. The lack of broadening also implies that NPLs 
somehow avoid the negative impact of Ostwald ripening.

Due to the many open questions, we have recently sought to clarify the NPL growth mechanism. Our efforts were initiated by a simple experiment that provided an important clue. ${ }^{40}$ Namely, we found that zincblende CdSe and CdS NPLs would form in simple solvent-free melts that contained only the chalcogen and a single cadmium carboxylate. This indicates (in agreement with other results) that neither a soft template nor selective binding of different ligands is necessary to obtain NPL growth.

After considering many possible mechanisms, we proposed a simple model based on a kinetic instability. ${ }^{40}$ We showed that under certain conditions, lateral growth on a narrow (few-monolayerthick) "side" facet of a NPL should be orders of magnitude faster than growth on the wide "top" or "bottom" facets. This can occur because the kinetic barrier for nucleation of an additional monolayer should be significantly reduced on a narrow facet. To be "narrow" the facet must have a dimension smaller than the critical island size for growth on a bulk surface of the same material. Indeed, this picture could immediately explain why CdE NPLs had been synthesized only up to a certain thickness. ${ }^{41-43}$ For NPLs with thicknesses comparable to or larger than the critical island size, lateral growth would have the same growth rate as that on the wide facets (i.e. on bulk surfaces). Isotropic growth would then be expected.

The above model could also produce specific quantitative predictions that were consistent with experiments. First, in the standard reactions containing only cadmium carboxylates and a $S$, $\mathrm{Se}$, or Te precursor, it could reproduce the specific thickness ranges obtained in syntheses of NPLs of CdS [ 2 to 5 monolayers (MLs $)^{41}$, $\mathrm{CdSe}\left(2\right.$ to $\left.5 \mathrm{MLs}^{41}\right)$, and CdTe ( 2 to $8 \mathrm{MLs}^{39,41,44}$ ). Second, it could explain why the same reactants could lead to either QDs or NPLs, depending on the conditions. The kinetic instability requires nanocrystal growth to be surface-reaction limited. If diffusion of reactants is limiting, spherical QDs should result. Therefore, a synthesis with the same precursors, e.g. cadmium myristate and elemental selenium, can yield QDs or NPLs depending on the local concentration of the precursors. NPLs grow if the reactants are highly concentrated, which is the case in the solvent-free melt. In the original CdSe NPL synthesis, ${ }^{17}$ a short-chain cadmium carboxylate was required to initiate the NPL growth. We have shown that this step causes the $\mathrm{Cd}$ precursor to phase separate as droplets from the organic solvent (octadecene). ${ }^{40}$ This concentrates the reactants.

To test the intrinsic-stability model further, we then considered NPL growth under conditions in which the reactants are depleted (i.e. the supersaturation of monomers is reduced). ${ }^{45}$ In this case, effects due to Ostwald ripening should arise. Because the standard theory of Ostwald ripening assumes particle shapes that are in thermodynamic equilibrium, ${ }^{7,45,46}$ this theory cannot be directly applied to the non-equilibrium shapes of NPLs. Thus, we developed a microscopic model that included the kinetics and thermodynamics of attachment and detachment of CdSe monomers to different facets of a NPL as a function of monomer concentration. ${ }^{45}$ Simulations revealed an interesting $2 \mathrm{D}$ Ostwald ripening process that included several important stages: (i) Early in the simulation, small isotropic nuclei of many different sizes appeared (e.g., cubes containing $2 \times 2 \times 2,3 \times 3 \times 3$, and $4 \times 4 \times 4$ monomers). Random attachment of additional monomers to these nuclei was sufficient to break symmetry and drive anisotropic growth via the kinetic instability. (ii) The first NPLs to appear out of this population of nuclei were the 2-ML-thick NPLs. 2-ML nuclei grow laterally the quickest because their narrow facets exhibit the fastest growth rate (compared to all other growth rates, including the narrow facets on thicker nuclei). (iii) As the monomers are consumed by NPL growth, 2D Ostwald ripening in the 2-ML NPL fraction is observed. 2-ML facets have the highest solubility compared to thicker facets. Moreover, within a population of 2-ML NPLs, the solubility of the narrow facet increases as the NPL becomes smaller laterally. Thus, as the monomer concentration decreases, the laterally small 2-ML NPLs will eventually become unstable and start to shrink, donating their material to laterally larger 2-ML NPLs, which remain stable and expand. This can be thought of as a $2 \mathrm{D}$ analog of the conventional Ostwald ripening. (iv) Eventually, the monomer concentration falls sufficiently that the narrow facets on all 2-ML NPLs become unstable, and this thickness population disappears. (v) The material is then transferred to the 3-ML-thick nuclei, which repeat the same sequence described above for the 2-ML NPLs. They expand, exhibit 2D Ostwald ripening, and eventually disappear. (vi) This process repeats sequentially for 4- and 5-ML NPLs.

These six stages describe our basic growth model. An important point is that it predicts that thicker NPLs appear without any growth on the wide facets. Growth (and dissolution) is only occurring on the narrow facets. We also note that the stages can overlap, causing several NPL thickness populations to coexist simultaneously. This is typically observed in real syntheses, in which 2 or more NPL thicknesses can be present at the same time, requiring post-synthetic selective precipitation to obtain a NPL sample of a single thickness. In our simulations a range of thicknesses existed throughout the growth in the form of small nuclei. However, the mass fraction of monomers contained in most of these thickness populations remained small until each cycled through its lateral growth and ripening process. This occurred sequentially from $m=2$ to 3 to 4 to 5 , with some coexistence of $m$ and $m+1$ as the mass fraction of the thinner NPL population decreased while that of the next thickness increased. ${ }^{45}$ These simulations could then potentially explain how a population of NPLs under prolonged heating can sequentially increase in thickness from $m$ to $m+1$ monolayers.

Here, our aim is to test the results of these simulations against careful ripening experiments. Specifically, we examine what happens when laterally small ("baby") CdS NPLs are heated. We find that such NPLs indeed grow laterally and, at sufficiently high temperatures, transform to thicker NPLs without the addition of any further reactants. This suggests that material must be transferred from smaller NPLs to larger ones. Indeed, we confirm this conclusion by heating laterally large CdSe NPLs in the presence of "baby" CdS NPLs. The result is CdSe-CdS core-crown NPLs, in which $\mathrm{CdS}$ has been transferred to the narrow faces of the preformed CdSe NPLs. These results support our previously proposed model and give further insight into the microscopic growth mechanism of NPLs.

\section{EXPERIMENTAL SECTION}

Chemicals. 1-octadecene (ODE, 90\%, \#O806), 2-propanol ( $\geq 99.9 \%$, \#34965), acetonitrile ( $\geq 99.9 \%, \# 34998)$, cadmium acetate dihydrate $\left[\mathrm{Cd}(\mathrm{OAc})_{2} \cdot 2 \mathrm{H}_{2} \mathrm{O}, 98 \%\right.$, \# 289159], oleic acid (OA, $90 \%$, \#364525), selenium ( $\geq 99.5 \%$, \#209651), toluene $(\geq 99.7 \%$, $\# 89681)$, trifluoroacetic acid $(\geq 99.0 \%, \# 302031)$, and trifluoroacetic anhydride ( $\geq 99 \%$, \#106232) were purchased from Sigma-Aldrich. Cadmium oxide (99.999\%, \#48-0800), triethylamine (99\%, \#157910010), $n$-hexane, absolute ethanol, and acetyl sulfide (>95.0\%, \#A0976) were bought from Strem Chemicals, Acros Organics, Thommen-Furler AG (Rüti b. Bern, Switzerland), alcosuisse (Bern, Switzerland), and Tokyo Chemical Industries, respectively. All chemicals were used as received without 
further purification.

Synthesis of Cadmium Oleate $\left[\mathrm{Cd}(\text { oleate })_{2}\right]$ and Cadmium Myristate $\left[\mathbf{C d}(\mathbf{m y r})_{2}\right]$. Cadmium carboxylates were synthesized according to a modified protocol by Hendricks et al. ${ }^{47}$ Briefly, $5.75 \mathrm{~g}$ of $\mathrm{CdO}$ was dispersed in $20 \mathrm{~mL}$ of acetonitrile. The mixture was stirred and cooled with a water bath at room temperature, and $0.7 \mathrm{~mL}$ of trifluoroacetic acid and $6.2 \mathrm{~mL}$ of trifluoroacetic anhydride were added. After $5 \mathrm{~min}$, the water bath was removed and the flask mildly heated until the cadmium oxide had dissolved. In a separate flask, $28.6 \mathrm{~mL}$ oleic acid (20.6 g myristic acid), $180 \mathrm{~mL}$ of isopropanol, and $14.0 \mathrm{~mL}$ of trimethylamine were mixed. The cadmium trifluoroacetate solution was added to the oleic acid (myristic acid) solution. The white precipitate was suction-filtered through a fritted glass funnel and rinsed thoroughly with methanol. The final product was dried in a vacuum oven at $40^{\circ} \mathrm{C}$ and stored under ambient conditions.

Synthesis of "Baby" CdS NPLs. $90 \mathrm{~mL}$ of ODE, $1.3 \mathrm{~mL}$ of OA, and $480 \mathrm{mg} \mathrm{Cd}(\mathrm{OAc})_{2} \cdot 2 \mathrm{H}_{2} \mathrm{O}$ were mixed in a $250 \mathrm{~mL} 4$-neck round-bottom flask. The mixture was heated to $120^{\circ} \mathrm{C}$ under vacuum and then degassed for $2 \mathrm{~h}$. Typically, the pressure in the flask dropped below 0.13 mbar. The flask was then flushed with $\mathrm{N}_{2}$, which caused the temperature to rise by a few degrees. Before proceeding, the temperature was re-stabilized at $120^{\circ} \mathrm{C} .0 .1 \mathrm{~mL}$ of acetyl sulfide was then swiftly injected and the reaction mixture heated to $180^{\circ} \mathrm{C}$. When $180^{\circ} \mathrm{C}$ was reached, the heating mantle was rapidly removed and the reaction mixture quenched in a water bath. The resulting "baby" NPLs were washed twice by precipitation with ethanol, centrifugation at $8000 \mathrm{rpm}(8590 \mathrm{~g})$ for $10 \mathrm{~min}$, and redispersion in $n$-hexane. The final product, which contains laterally small 2- and 3ML CdS NPLs, was redispersed in $60 \mathrm{~mL}$ ODE or $60 \mathrm{~mL}$ toluene for the ripening or thin-film experiments, respectively.

Synthesis of 4-ML CdSe NPLs. 4-ML-thick CdSe NPLs were synthesized following an adapted protocol from Pedetti et al. ${ }^{48}$ Briefly, $24 \mathrm{mg}$ of Se, $340 \mathrm{mg}$ of $\mathrm{Cd}(\mathrm{myr})_{2}$, and $30 \mathrm{~mL}$ of ODE were mixed in a $100 \mathrm{~mL}$ three-neck flask and degassed for $30 \mathrm{~min}$ at room temperature. The flask was then purged with $\mathrm{N}_{2}$ and the temperature set to $240^{\circ} \mathrm{C}$. When the temperature reached $205^{\circ} \mathrm{C}, 86 \mathrm{mg}$ of $\mathrm{Cd}(\mathrm{OAc})_{2} \cdot 2 \mathrm{H}_{2} \mathrm{O}$ was added to the flask. $10 \mathrm{~min}$ after the temperature reached $240^{\circ} \mathrm{C}$, the reaction was rapidly quenched in an ice bath. When the temperature reached room temperature, $2 \mathrm{~mL}$ of $\mathrm{OA}$ and $30 \mathrm{~mL}$ of $n$-hexane were injected to the reaction mixture. After centrifugation at $6000 \mathrm{rpm}(4830 \mathrm{~g})$ for $10 \mathrm{~min}$, the supernatant was discarded and the precipitate redispersed in $20 \mathrm{~mL}$ $n$-hexane.

Ripening Experiments. $8 \mathrm{~mL}$ of the "baby" CdS NPL dispersion, $22 \mathrm{~mL}$ of $\mathrm{ODE}$, and $35 \mathrm{mg}$ of $\mathrm{Cd}(\mathrm{OAc})_{2} \cdot 2 \mathrm{H}_{2} \mathrm{O}$ were mixed in a $50 \mathrm{~mL}$ three-neck flask. The mixture was degassed under vacuum at $120^{\circ} \mathrm{C}$ for $60 \mathrm{~min}$ and then purged with $\mathrm{N}_{2}$. The temperature was ramped to the desired temperature $\left(160-190^{\circ} \mathrm{C}\right)$, held for $12 \mathrm{~h}$ $50 \mathrm{~min}$, and then cooled to room temperature naturally. The reaction mixture was diluted with $30 \mathrm{~mL}$ of $n$-hexane and centrifuged at $3000 \mathrm{rpm}(1210 \mathrm{~g})$ for $5 \mathrm{~min}$ to remove left-over $\mathrm{Cd}(\mathrm{OAc})_{2}$. The NPLs remained in the supernatant and were purified twice by addition of ethanol, centrifugation at $4000 \mathrm{rpm}$ $(2150 \mathrm{~g})$, and redispersion in $n$-hexane.

Synthesis of Thin Films. $130 \mathrm{mg} \mathrm{Cd}$ (oleate) ${ }_{2}$ was dissolved in $2 \mathrm{~mL}$ of the "baby" CdS NPL dispersion. $150 \mu \mathrm{L}$ of this dispersion was dropped on a glass slide and spun for $1 \mathrm{~min}$ at $800 \mathrm{rpm}$. The glass slide was then let dry for at least $30 \mathrm{~s}$. This procedure was repeated a total of four times in air. For transmission electron microscopy
(TEM), a $\mathrm{SiO}_{2}$-coated silicon nitride grid (Ted Pella, \#21531-10) was fixed on a glass slide with Kapton ${ }^{\oplus}$ tape and spun at $3500 \mathrm{rpm}$. While spinning, $10 \mu \mathrm{L}$ of the same dispersion was dropped on the grid. This procedure was repeated a total of three times in air. The thin films were then transferred into a $\mathrm{N}_{2}$-filled glovebox, put on a pre-heated hot plate $\left(\right.$ at $180^{\circ} \mathrm{C}$ or $200^{\circ} \mathrm{C}$ ) for $17 \mathrm{~h} 15 \mathrm{~min}$, and then allowed to cool naturally.

Transmission Electron Microscopy (TEM). Samples were prepared by drop casting dilute dispersions in $n$-hexane onto carboncoated copper grids. TEM micrographs were recorded on a Philips CM12 (operated at $100 \mathrm{kV}$ ). Energy dispersive X-ray spectroscopy (EDX) for elemental mapping was performed on an FEI Talos F200X (operated at $200 \mathrm{kV}$ ).

Spectroscopy. Aliquots were diluted in $n$-hexane and transferred to quartz cuvettes. Absorption spectra were collected on a Varian Cary 50 spectrometer and photoluminescence (PL) spectra on an Edinburgh Instruments FLS 980 fluorometer.

\section{RESULTS AND DISCUSSION}

"Baby" CdS NPLs. Because we had previously identified acyl chalcogenides as intermediates that form when cadmium chalcogenides are synthesized from a cadmium carboxylate and elemental chalcogen, ${ }^{49,50}$ we decided to utilize acetyl sulfide directly as the chalcogenide precursor in our NPL reactions. We modified a previously published NPL protocol ${ }^{29}$ by replacing selenium with acetyl sulfide. Following the same temperature profile [ramping to $240{ }^{\circ} \mathrm{C}$ with addition of $\mathrm{Cd}(\mathrm{OAc})_{2} \cdot \mathrm{H}_{2} \mathrm{O}$ at $\left.205^{\circ} \mathrm{C}\right]$ we obtained 4 ML-thick CdS NPLs with a prominent absorption peak at $406 \mathrm{~nm} .{ }^{41}$ The lateral size of the resulting NPLs was approximately $10 \times 20 \mathrm{~nm}$, as determined by TEM (see Section $S 1$ in the Supporting Information). This result just confirms the now expected result that acetyl sulfide can be used to prepare conventional CdS NPLs.

More interesting, the use of acetyl sulfide as the chalcogenide source also allowed us to track the reaction product during the temperature ramp. We exploited this to analyze the growth. Namely, we collected aliquots and measured optical absorption spectra and TEM micrographs on each. Immediately after the injection of acetyl sulfide at $120^{\circ} \mathrm{C}$, a sharp peak at $321 \mathrm{~nm}$ appeared (Figure 1a), and small crystallites could be observed in TEM (Figure 1b). This spectral feature, which disappears upon further heating, was previously assigned to a magic-sized cluster (MSC). ${ }^{51,52}$ A definitive assignment of this species is challenging due to its small size.

With further heating to $130^{\circ} \mathrm{C}$, two new absorption peaks emerged at 326 and $359 \mathrm{~nm}$. The corresponding TEM micrographs (Figure 1c) show mostly very thin needle-like structures but also some particles that are more isotropic in the lateral dimensions. With increasing temperature to 150 and then $160{ }^{\circ} \mathrm{C}$ (Figure 1a), the peak at $326 \mathrm{~nm}$ grew, the peak at $359 \mathrm{~nm}$ gradually vanished (see Figure S2 in the Supporting Information to support that it is vanishing rather than just shifting), and a new sharp feature at $377 \mathrm{~nm}$ appeared. This latter peak has previously been attributed to another CdS MSC..$^{53}$ However, it is also possible that the 359 and $377 \mathrm{~nm}$ features arise from the needle-like structures. These two absorption peaks disappeared (Figure 1a) together with the needle-like structures (Figure 1d,e) in the $180^{\circ} \mathrm{C}$ aliquot.

The remaining two absorption maxima in Figure 1a at 326 and $371 \mathrm{~nm}$ appear to arise independently. From this, we conclude that two distinct species are present. However, we could not detect obvious differences between the nanocrystals in the TEM micrographs (Figure 1d,e), other than small and apparently random 

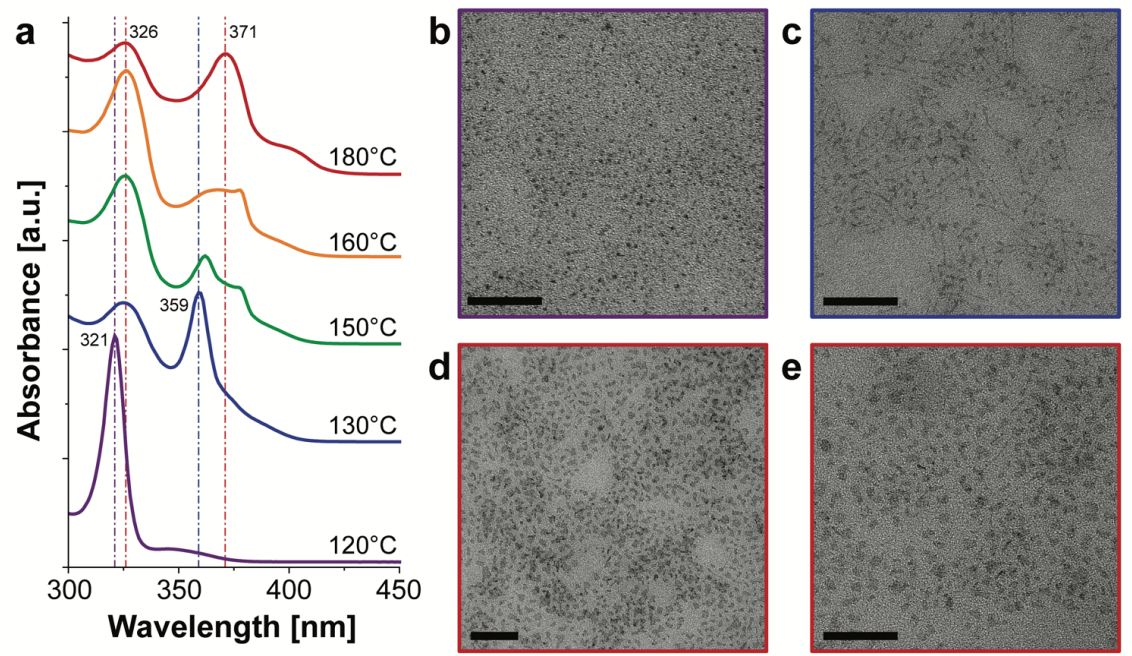

Figure 1. Synthesis of "baby" CdS NPLs with Cd(oleate) ${ }_{x}(\mathrm{OAc})_{2-\mathrm{x}}$ and acetyl sulfide. (a) Absorption spectra of aliquots taken as the temperature is increased after injection of acetyl sulfide. Vertical lines at 321, 326, 359, and $371 \mathrm{~nm}$ are provided to compare the spectra. TEM micrographs for aliquots taken: (b) at $120^{\circ} \mathrm{C}$ immediately after the injection of acetyl sulfide, (c) at $130^{\circ} \mathrm{C},(\mathrm{d})$ at $180^{\circ} \mathrm{C}$ at low magnification, and (e) at $180^{\circ} \mathrm{C}$ at higher magnification. Scale bars are $50 \mathrm{~nm}$.
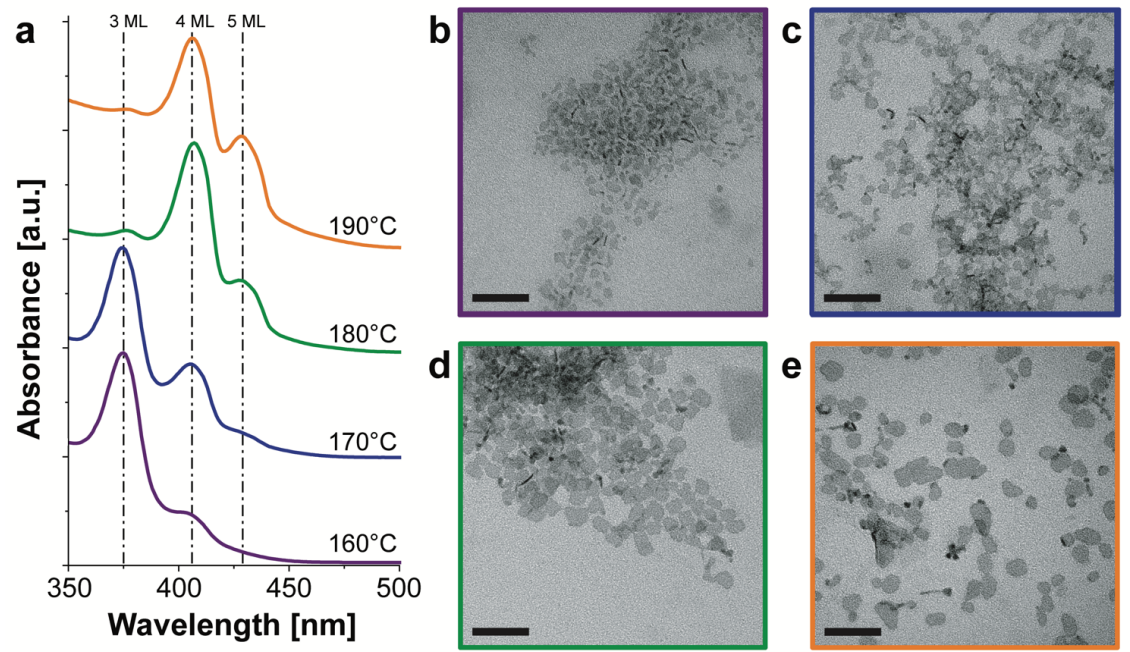

Figure 2. Ostwald ripening of "baby" CdS NPLs. (a) Absorption spectra of the CdS NPLs formed as a function of temperature. Corresponding TEM micrographs of the NPLs formed at (b) $160^{\circ} \mathrm{C}$, (c) $170^{\circ} \mathrm{C}$, (d) $180^{\circ} \mathrm{C}$, and (e) $190^{\circ} \mathrm{C}$. Scale bars are $50 \mathrm{~nm}$.

variations in the lateral shape and size. Both absorption maxima occur at wavelengths very close to literature values for the electron/light-hole transitions for 2- and 3-ML CdS NPLs, ${ }^{41}$ with a small blue-shift ( 326 vs. $331 \mathrm{~nm}$ and 371 vs. $373 \mathrm{~nm}$, respectively). Blue-shifts in the excitonic transitions of CdSe NPLs have been observed when their lateral sizes were small. ${ }^{18}$ Thus, our observations can be explained by the presence of 2- and 3-ML CdS NPLs that are laterally quantum-confined ("baby" CdS NPLs). This conclusion is also consistent with the small nanocrystals that appear in Figure 1d,e.

When the same reaction was conducted at constant temperature, the same evolution was observed. In other words, after degassing we heated the reaction mixture to a certain temperature $(140,160$, or $180^{\circ} \mathrm{C}$ ), and then the injection and growth were performed at this temperature. Higher temperatures only increased the reaction rate (see Section S2 in the Supporting Information).

Ostwald Ripening. Based on the results from the previous section, we prepared "baby" CdS NPLs by quenching the reaction at $180^{\circ} \mathrm{C}$ and precipitating the product twice with ethanol. To investigate Ostwald ripening of these NPLs, we then dispersed them in $\mathrm{ODE}$ with $\mathrm{Cd}(\mathrm{OAc})_{2} \cdot 2 \mathrm{H}_{2} \mathrm{O}$ and heated them at different constant temperatures. Even though no sulfide precursor was added, we observed lateral growth and transitions to thicker populations, depending on the temperature. In agreement with the model discussed above, the thinnest $(2 \mathrm{ML})$ population was the most unstable, and it rapidly dissolved completely even at relatively low temperatures $\left(<160^{\circ} \mathrm{C}\right)$, as evidenced by the disappearance of the absorption maximum at $326 \mathrm{~nm}$. At temperatures above $160^{\circ} \mathrm{C}$ (Figure 2a), we observed a continuous transition from populations with thicknesses of $3 \mathrm{ML}$ (electron/light-hole transition at $374 \mathrm{~nm}$ ) to $4 \mathrm{ML}(407 \mathrm{~nm})$ and then $5 \mathrm{ML}(429 \mathrm{~nm})$. While the absorption spectra show different thicknesses of NPLs present at each investigated temperature, the NPLs with different thicknesses could not be distinguished by TEM (Figure $2 b-e$ ). The average lateral size of the NPLs increases with temperature, and the lateral size distribution is clearly broad.

When the same ripening experiment was conducted without additional short-chain cadmium carboxylate, i.e. "baby" CdS NPLs 
were heated in $\mathrm{ODE}$ without $\mathrm{Cd}(\mathrm{OAc})_{2}$, the characteristic absorption peaks (at 374 and $407 \mathrm{~nm}$ ) disappeared, and featureless absorption grew in at wavelengths longer than $450 \mathrm{~nm}$. This suggests the formation of crystals with random sizes and shapes (see Section S3 in the Supporting Information). This observation is consistent with the prediction that NPL growth requires surface-reactionlimited conditions, which is presumably not maintained here. ${ }^{46}$ Without the short-chain $\mathrm{Cd}(\mathrm{OAc})_{2}$, phase-segregated droplets do not form in the ODE. ${ }^{40,45}$ Thus, any CdS monomers released by dissolving "baby" NPLs are not concentrated within these droplets, and conditions for $2 \mathrm{D}$ growth are not satisfied.

When laterally extended 4-ML CdS NPLs were kept at high temperatures in the presence of $\mathrm{Cd}(\mathrm{OAc})_{2}$, no transitions to thicker populations were observed even after several weeks (see Section S4 in the Supporting Information). This is an important observation for the mechanism behind the transition to thicker NPL populations. It had been hypothesized that the transformation of $m$ to $m+1$ MLs for NPLs happens because of surface nucleation and rapid overgrowth of additional layers on the wide "top" and "bottom" facets. ${ }^{38}$ The lack of experimentally observed thickness transitions for the 4-ML NPLs is inconsistent with this hypothesis. Moreover, it would be difficult to explain why such a process would stop at $m+1$ MLs. Rather, growth on the wide facets would presumably lead to a broad range of thicknesses.

By contrast, our kinetic-instability model predicts that growth on the wide facets is highly unlikely compared to lateral growth on the narrow facets. Even if the thermal energy (i.e. the temperature) were increased such that the kinetic barrier for growth on the wide facets could be overcome, this would lead to isotropic growth. Under these conditions, the kinetic instability that drives NPL growth would disappear. Our model also predicts that the dissolution of NPLs should be easier if they are laterally small. Thus, the transformation to thicker populations should be faster for "baby" NPLs, as observed. Laterally extended NPLs can be stable indefinitely depending on the monomer concentration. Therefore, our results agree with the model predictions that transitions between different NPL thicknesses occur via lateral dissolution and growth.

Nanoheterostructures. If correct, the growth model above should presumably be valid for any cubic semiconductor that forms NPLs. Therefore, it should be possible to combine materials and form nanoheterostructures such as core-crown NPLs using Ostwald ripening. To test this, we synthesized 4-ML CdSe NPLs with a lateral size of approximately $10 \times 20 \mathrm{~nm}$ according to a published protocol. $^{48}$ We then dispersed these NPLs with $\mathrm{Cd}(\mathrm{OAc})_{2} \cdot 2 \mathrm{H}_{2} \mathrm{O}$ and "baby" CdS NPLs and heated the mixture to $160{ }^{\circ} \mathrm{C}$. The concentrations and temperatures corresponded to the Ostwald ripening experiments for $\mathrm{CdS}$ described above, but with the additional presence of CdSe NPLs.

The experiments described in the previous section on 4-ML CdS NPLs suggest that the laterally extended 4-ML CdSe NPLs should be stable (i.e. not dissolving) under the reaction conditions. The "baby" CdS NPLs should also undergo the same 3- to 4-ML thickness transformation as observed without the CdSe NPLs. However, because the CdSe NPLs have the same thickness as the product of the CdS transformation, heterogeneous nucleation of $\mathrm{CdS}$ on the narrow facets of the CdSe NPLs could also occur. Thus, we might expect the final product to be a mixture of 4-ML CdS NPLs and 4-ML CdSe-CdS core-crown NPLs. We stress that the goal of these experiments is not to improve synthetic approaches to $\mathrm{CdSe}-$ CdS core-crown NPLs, but rather to test our growth model.
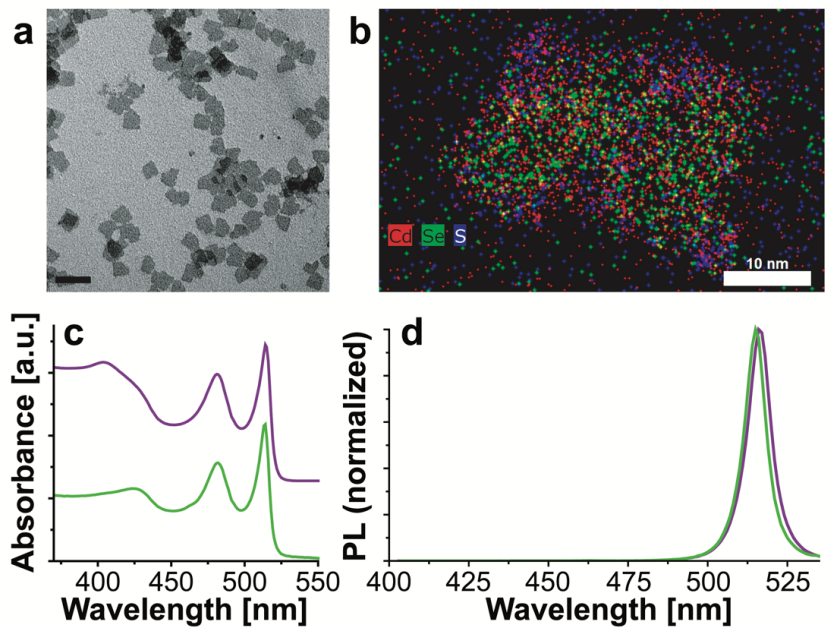

Figure 3. Nanostructures formed by heating a mixture of 4-ML CdSe NPLs and "baby" CdS NPLs. (a) TEM micrograph of the NPLs that result after ripening. Scale bar is $40 \mathrm{~nm}$. (b) EDX mapping (red $=\mathrm{Cd}$, green $=\mathrm{Se}$, and blue $=\mathrm{S}$ ) of two NPLs lying next to each other. Collecting significant signal was challenging due to electron-beam damage. (c) Absorption spectra of the CdSe NPLs before (green) and after (purple) ripening. The product shown after ripening is purified. Further details and data are shown in Section S5 in the Supporting Information. (d) Normalized photoluminescence (PL) spectra of the CdSe NPLs before (green) and after (purple) ripening. Excitation is at $400 \mathrm{~nm}$. A $\sim 1 \mathrm{~nm}$ peak shift is observed. The above results indicate the formation of $\mathrm{CdSe}-\mathrm{CdS}$ core-crown NPLs during the ripening process.

Absorption spectra of aliquots taken during the reaction do show the transformation of 3- to 4-ML CdS NPLs. Simultaneously, the electron/light-hole and electron/heavy-hole transitions of the CdSe NPLs remain unchanged (Section S5 in the Supporting Information). By adding small amounts of ethanol to the dispersion, we could selectively precipitate a portion of pure core-crown NPLs. The rest remained in the supernatant along with the 4-ML CdS NPLs. The final, washed precipitate was analyzed by TEM (Figure 3a), EDX (Figure 3b), and optical spectroscopy (Figure 3c,d).

EDX was challenging to collect due to electron-beam sensitivity of the NPLs. This explains the low signal in Figure 3b. Nevertheless, the EDX image, which contains two adjacent NPLs, suggests an accumulation of CdS around the edges of the NPLs. The Se signal appears more in the NPL cores. This suggests that 4-ML CdSe-CdS core-crown NPLs were formed.

This conclusion is also supported by the absorption spectra of the CdSe NPLs before and after heating, which are almost identical except for the appearance of a small local maximum at $405 \mathrm{~nm}$ (Figure 3c). This feature, which coincides with the electron/lighthole transition of 4-ML CdS NPLs, also appears in the PL excitation spectrum obtained by monitoring the emission of the CdSe NPLs at $520 \mathrm{~nm}$ (Section S5 in the Supporting Information). This indicates that the CdS is co-located with the CdSe NPLs. Finally, the emission intensity maximum of the CdSe NPLs was slightly red-shifted after ripening; no emission from 4-ML CdS NPLs was detected (Figure $3 d)$. All of these data are consistent with prior measurements on $\mathrm{CdSe}-\mathrm{CdS}$ core-crown NPLs. ${ }^{54}$ Thus, these results indicate that the "baby" CdS NPLs dissolved and the material grew on the 4-ML CdSe NPLs. We note that we also attempted to ripen "baby" CdSe NPLs in the presence of CdS NPLs to form CdS-CdSe core-crown NPLs. However, we have not yet found conditions (concentration, temperature, etc.) where the "baby" CdSe NPLs would dissolve 


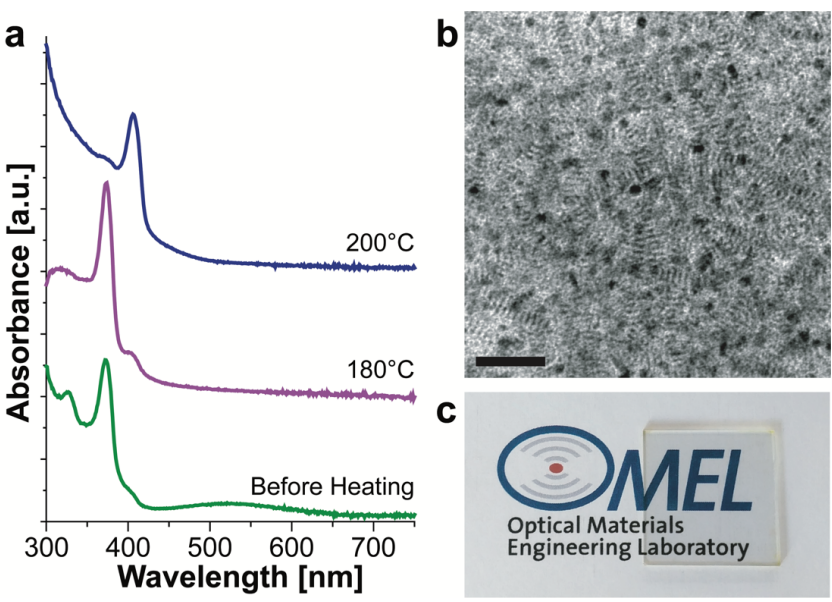

Figure 4. Ripening of thin films containing "baby" CdS NPLs and $\mathrm{Cd}$ (oleate) $)_{2}$ deposited on glass substrates. (a) Absorption spectra of films before heating and after heating to either $180^{\circ} \mathrm{C}$ or $200^{\circ} \mathrm{C}$. (b) TEM micrograph and (c) photograph of the final film produced. Scale bar is $50 \mathrm{~nm}$. (The OMEL logo, designed by D. Norris, is used with permission.)

while the CdS NPLs were stable.

Thin Films. Our growth model also suggests that the same Ostwald ripening will occur in a thin-film matrix that contains only "baby" CdS NPLs and cadmium carboxylate. Such expectations follow directly from our previous work on the formation of NPLs in solvent-free melts. ${ }^{40}$ Therefore, we dispersed "baby" CdS NPLs with $\mathrm{Cd}$ (oleate $)_{2}$ in toluene and spin coated the mixture onto glass slides or TEM grids. After the toluene evaporated a thin solid film was formed. These substrates were then heated above the melting temperature of $\mathrm{Cd}(\text { oleate })_{2}$. Because the melt is then essentially a highly concentrated liquid dispersion of "baby" NPLs, CdS monomers released during ripening have easy access to neighboring NPLs. Consequently, we would expect surface-reaction-limited conditions to exist. Consistent with this, when our films were heated to $180^{\circ} \mathrm{C}$ and $200^{\circ} \mathrm{C}$ absorption spectra (Figure 4a) revealed the same transformation to thicker NPLs that were observed in liquid dispersions (Figure 2).

Presumably due to the high concentration of the resulting NPLs, they also formed stacks with lengths in the range of $100 \mathrm{~nm}$, as observed in TEM (Figure 4b). While more research is necessary to understand the observed stacking, recent publications have shown that CdSe NPLs form large stacks in the presence of oleic acid due to the depletion interaction. ${ }^{55} \mathrm{~A}$ similar mechanism may be responsible here. We also note that thin films prepared with this protocol were transparent and smooth (Figure 4c).

\section{CONCLUSIONS}

We have performed a series of experiments that indicate that "baby" CdS NPLs undergo two-dimensional Ostwald ripening under surface-reaction-limited conditions. These results further support our previously published NPL growth model. In particular, they demonstrate that thickness transformations occur via dissolution of thinner populations. The dissolved material is then redeposited on thicker NPLs through lateral growth. Our observation of temperature-dependent thickness transitions and of CdSe-CdS core-crown NPLs are inconsistent with both layer overgrowth on the wide facet ${ }^{18}$ and oriented attachment. ${ }^{56}$ Instead, they support the theoretical prediction that only lateral growth is operable, with the thicker NPLs growing at the expense of thinner ones. ${ }^{45}$
Because NPLs can be grown via Ostwald ripening without being restricted by the kinetics or thermodynamics of side reactions (e.g. reduction of the chalcogen), we believe that future studies on this system with other materials can be useful for providing quantitative data on different NPLs, such as growth temperatures, accessible thicknesses, or whether two-dimensional growth can occur at all. Furthermore, it can potentially enable the synthesis of nanoheterostructures in systems where this is currently not possible, such as the metal halide perovskites.

\section{ASSOCIATED CONTENT Supporting Information}

Further synthetic details and experimental data, including absorption, PL, and PL excitation spectra, and TEM micrographs.

\section{AUTHOR INFORMATION \\ Corresponding Author \\ *E-mail: dnorris@ethz.ch}

\section{ORCID}

Philippe N. Knüsel: 0000-0002-9546-5809

Andreas Riedinger: 0000-0002-7732-0606

Aurelio Rossinelli: 0000-0001-6930-4190

Florian D. Ott: 0000-0002-4979-7554

Aniket Mule: 0000-0001-8387-080X

David J. Norris: 0000-0002-3765-0678

\section{Notes}

The authors declare no competing financial interest.

\section{ACKNOWLEDGMENTS}

This work was supported by ETH Research Grant ETH-38 14-1, by the Swiss National Science Foundation under Grant No. 200021-165559, and by the European Research Council under the European Union's Seventh Framework Program (FP/2007-2013) / ERC Grant Agreement Nr. 339905 (QuaDoPS Advanced Grant). We thank F. Starsich for assistance with XRD measurements, C. Zaubitzer for help with TEM imaging, J. Cui for fruitful discussions and critical reading of the manuscript, and S. Meyer for technical assistance. We benefitted from the use of facilities at the Scientific Center for Optical and Electron Microscopy (ScopeM) at ETH Zurich.

\section{REFERENCES}

(1) Murray, C. B.; Kagan, C. R.; Bawendi, M. G., Synthesis and Characterization of Monodisperse Nanocrystals and Close-Packed Nanocrystal Assemblies. Annu. Rev. Mater. Sci. 2000, 30, 545-610.

(2) Zhuang, Z. B.; Peng, Q.; Li, Y. D., Controlled Synthesis of Semiconductor Nanostructures in the Liquid Phase. Chem. Soc. Rev. 2011, 40, 5492-5513.

(3) Klimov, V. I., Nanocrystal Quantum Dots. 2nd ed.; CRC Press: Boca Raton, 2010.

(4) Efros, Al. L.; Efros, A. L., Interband Absorption of Light in a Semiconductor Sphere. Sov. Phys. Semicond. 1982, 16, 772-775.

(5) Brus, L. E., A Simple Model for the Ionization Potential, Electron Affinity, and Aqueous Redox Potentials of Small Semiconductor Crystallites. J. Chem. Phys. 1983, 79, 5566-5571.

(6) Brus, L. E., Electron-Electron and Electron-Hole interactions in Small Semiconductor Crystallites: The Size Dependence of the Lowest Excited Electronic State. J. Chem. Phys. 1984, 80, 4403-4409.

(7) Ostwald, W., Über die vermeintliche Isomerie des roten und gelben Quecksilberoxyds und die Oberflächenspannung fester Körper. Z. Phys. Chem. 1900, 34, 495-503. 
(8) Peng, X.; Wickham, J.; Alivisatos, A. P., Kinetics of II-VI and III-V Colloidal Semiconductor Nanocrystal Growth: 'Focusing' of Size Distributions. J. Am. Chem. Soc. 1998, 120, 5343-5344.

(9) Peng, X.; Manna, L.; Yang, W.; Wickham, J.; Scher, E.; Kadavanich, A.; Alivisatos, A. P., Shape Control of CdSe Nanocrystals. Nature 2000, 404 59-61.

(10) Holmes, J. D.; Johnston, K. P.; Doty, R. C.; Korgel, B. A., Control of Thickness and Orientation of Solution-Grown Silicon Nanowires. Science 2000, 287, 1471-1473.

(11) Yu, H.; Li, J. B.; Loomis, R. A.; Wang, L. W.; Buhro, W. E., TwoVersus Three-Dimensional Quantum Confinement in Indium Phosphide Wires and Dots. Nat. Mater. 2003, 2, 517-520.

(12) Grebinski, J. W.; Hull, K. L.; Zhang, J.; Kosel, T. H.; Kuno, M., Solution-Based Straight and Branched CdSe Nanowires. Chem. Mater. 2004, 16, 5260-5272.

(13) Manna, L.; Scher, E. C.; Alivisatos, A. P., Synthesis of Soluble and Processable Rod-, Arrow-, and Teardrop-, and Tetrapod-Shaped CdSe Nanocrystals. J. Am. Chem. Soc. 2000, 122, 12700-12706.

(14) Manna, L.; Milliron, D. J.; Meisel, A.; Scher, E. C.; Alivisatos, A. P., Controlled Growth of Tetrapod-Branched Inorganic Nanocrystals. Nat. Mater. 2003, 2, 382-385.

(15) Milliron, D. J.; Hughes, S. M.; Cui, Y.; Manna, L.; Li, J.; Wang, L.W.; Alivisatos, A. P., Colloidal Nanocrystal Heterostructures with Linear and Branched Topology. Nature 2004, 430, 190-195.

(16) Liu, L.; Zhuang, Z.; Xie, T.; Wang, Y.-G.; Li, J.; Peng, Q.; Li, Y., Shape Control of CdSe Nanocrystals with Zinc Blende Structure. J. Am. Chem. Soc. 2009, 131, 16423-16429.

(17) Ithurria, S.; Dubertret, B., Quasi 2D Colloidal CdSe Platelets with Thicknesses Controlled at the Atomic Level. J. Am. Chem. Soc. 2008, 130, 16504-16505

(18) Nasilowski, M.; Mahler, B.; Lhuillier, E.; Ithurria, S.; Dubertret, B., Two-Dimensional Colloidal Nanocrystals. Chem. Rev. 2016, 116, 10934 10982 .

(19) Yeltik, A.; Delikanli, S.; Olutas, M.; Kelestemur, Y.; Guzelturk, B.; Demir, H. V., Experimental Determination of the Absorption Cross-Section and Molar Extinction Coefficient of Colloidal CdSe Nanoplatelets. J. Phys. Chem. C 2015, 119, 26768-26775.

(20) Tessier, M. D.; Javaux, C.; Maksimovic, I.; Loriette, V.; Dubertret, B., Spectroscopy of Single CdSe Nanoplatelets. ACS Nano 2012, 6, 67516758.

(21) Scott, R.; Achtstein, A. W.; Prudnikau, A. V.; Antanovich, A.; Siebbeles, L. D. A.; Artemyev, M.; Woggon, U., Time-Resolved Stark Spectroscopy in CdSe Nanoplatelets: Exciton Binding Energy, Polarizability, and Field-Dependent Radiative Rates. Nano Lett. 2016, 16, 6576-6583.

(22) Chen, Z.; Nadal, B.; Mahler, B.; Aubin, H.; Dubertret, B., Quasi-2D Colloidal Semiconductor Nanoplatelets for Narrow Electroluminescence. Adv. Funct. Mater. 2014, 24, 295-302.

(23) She, C.; Fedin, I.; Dolzhnikov, D. S.; Demortière, A.; Schaller, R D.; Pelton, M.; Talapin, D. V., Low-Threshold Stimulated Emission Using Colloidal Quantum Wells. Nano Lett. 2014, 14, 2772-2777.

(24) Bertrand, G. H. V.; Polovitsyn, A.; Christodoulou, S.; Khan, A. H.; Moreels, I., Shape Control of Zincblende CdSe Nanoplatelets. Chem. Commun. 2016, 52, 11975-11978.

(25) Bouet, C.; Laufer, D.; Mahler, B.; Nadal, B.; Heuclin, H.; Pedetti, S.; Patriarche, G.; Dubertret, B., Synthesis of Zinc and Lead Chalcogenide Core and Core/Shell Nanoplatelets Using Sequential Cation Exchange Reactions. Chem. Mater. 2014, 26, 3002-3008.

(26) Izquierdo, E.; Robin, A.; Keuleyan, S.; Lequeux, N.; Lhuillier, E.; Ithurria, S., Strongly Confined HgTe 2D Nanoplatelets as Narrow NearInfrared Emitters. J. Am. Chem. Soc. 2016, 138, 10496-10501.

(27) Prudnikau, A.; Chuvilin, A.; Artemyev, M., CdSe-CdS Nanoheteroplatelets with Efficient Photoexcitation of Central CdSe Region through Epitaxially Grown CdS Wings. J. Am. Chem. Soc. 2013, 135, 14476 14479 .

(28) Ithurria, S.; Talapin, D. V., Colloidal Atomic Layer Deposition (cALD) using Self-Limiting Reactions at Nanocrystal Surface Coupled to
Phase Transfer between Polar and Nonpolar Media. J. Am. Chem. Soc. 2012 134, 18585-18590.

(29) Mahler, B.; Nadal, B.; Bouet, C.; Patriarche, G.; Dubertret, B., Core/Shell Colloidal Semiconductor Nanoplatelets. J. Am. Chem. Soc. 2012, 134, 18591-18598.

(30) Rossinelli, A. A.; Riedinger, A.; Marques-Gallego, P.; Knusel, P. N.; Antolinez, F. V.; Norris, D. J., High-Temperature Growth of Thick-Shell CdSe/CdS Core/Shell Nanoplatelets. Chem. Commun. 2017, 53, 99389941.

(31) Joo, J.; Son, J. S.; Kwon, S. G.; Yu, J. H.; Hyeon, T., LowTemperature Solution-Phase Synthesis of Quantum Well Structured CdSe Nanoribbons. J. Am. Chem. Soc. 2006, 128, 5632-5633.

(32) Schliehe, C.; Juarez, B. H.; Pelletier, M.; Jander, S.; Greshnykh, D.; Nagel, M.; Meyer, A.; Foerster, S.; Kornowski, A.; Klinke, C.; Weller, H., Ultrathin $\mathrm{PbS}$ Sheets by Two-Dimensional Oriented Attachment. Science 2010, 329, 550-553.

(33) Liu, Y.-H.; Wang, F.; Wang, Y.; Gibbons, P. C.; Buhro, W. E., Lamellar Assembly of Cadmium Selenide Nanoclusters into Quantum Belts. J. Am. Chem. Soc. 2011, 133, 17005-17013.

(34) Olutas, M.; Guzelturk, B.; Kelestemur, Y.; Yeltik, A.; Delikanli, S.; Demir, H. V., Lateral Size-Dependent Spontaneous and Stimulated Emission Properties in Colloidal CdSe Nanoplatelets. ACS Nano 2015, 9, 5041-5050.

(35) Ithurria, S.; Bousquet, G.; Dubertret, B., Continuous Transition from $3 \mathrm{D}$ to $1 \mathrm{D}$ Confinement Observed During the Formation of CdSe Nanoplatelets. J. Am. Chem. Soc. 2011, 133, 3070-3077.

(36) Bouet, C.; Mahler, B.; Nadal, B.; Abécassis, B.; Tessier, M. D.; Ithurria, S.; Xu, X.; Dubertret, B., Two-Dimensional Growth of CdSe Nanocrystals, from Nanoplatelets to Nanosheets. Chem. Mater. 2013, 25, 639-645.

(37) Li, Z.; Peng, X., Size/Shape-Controlled Synthesis of Colloidal CdSe Quantum Disks: Ligand and Temperature Effects. J. Am. Chem. Soc 2011, 133, 6578-6586.

(38) Li, Z.; Qin, H.; Guzun, D.; Benamara, M.; Salamo, G.; Peng, X., Uniform Thickness and Colloidal-Stable CdS Quantum Disks with Tunable Thickness: Synthesis and Properties. Nano Res. 2012, 5, 337-351.

(39) Pedetti, S.; Nadal, B.; Lhuillier, E.; Mahler, B.; Bouet, C.; Abécassis, B.; Xu, X.; Dubertret, B., Optimized Synthesis of CdTe Nanoplatelets and Photoresponse of CdTe Nanoplatelets Films. Chem. Mater. 2013, 25, 2455 2462.

(40) Riedinger, A.; Ott, F. D.; Mule, A.; Mazzotti, S.; Knusel, P. N. Kress, S. J. P.; Prins, F.; Erwin, S. C.; Norris, D. J., An Intrinsic Growth Instability in Isotropic Materials Leads to Quasi-Two-Dimensional Nanoplatelets. Nat. Mater. 2017, 16, 743-748.

(41) Ithurria, S.; Tessier, M. D.; Mahler, B.; Lobo, R. P. S. M.; Dubertret, B.; Efros, A. L., Colloidal Nanoplatelets with Two-Dimensional Electronic Structure. Nat. Mater. 2011, 10, 936-941.

(42) Christodoulou, S.; Climente, J. I.; Planelles, J.; Brescia, R.; Prato, M.; Martín-García, B.; Khan, A. H.; Moreels, I., Chloride-Induced Thickness Control in CdSe Nanoplatelets. Nano Lett. 2018, 18, 6248-6254.

(43) Cho, W.; Kim, S.; Coropceanu, I.; Srivastava, V.; Diroll, B. T.; Hazarika, A.; Fedin, I.; Galli, G.; Schaller, R. D.; Talapin, D. V., Direct Synthesis of Six-Monolayer $(1.9 \mathrm{~nm})$ Thick Zinc-Blende CdSe Nanoplatelets Emitting at 585 nm. Chem. Mater. 2018, 30, 6957-6960.

(44) Scott, R.; Kickhofel, S.; Schoeps, O.; Antanovich, A.; Prudnikau, A.; Chuvilin, A.; Woggon, U.; Artemyev, M.; Achtstein, A. W., Temperature Dependent Radiative and Non-Radiative recombination Dynamics in CdSe-CdTe and CdTe-CdSe Type II Hetero Nanoplatelets. Phys. Chem. Chem. Phys. 2016, 18, 3197-3203.

(45) Ott, F. D.; Riedinger, A.; Ochsenbein, D. R.; Knuesel, P. N.; Erwin S. C.; Mazzotti, M.; Norris, D. J., Ripening of Semiconductor Nanoplatelets. Nano Lett. 2017, 17, 6870-6877.

(46) Wagner, C., Theorie der Alterung von Niederschlägen durch Umlösen (Ostwald-Reifung). Z. Elektrochem. 1961, 65, 581-591.

(47) Hendricks, M. P.; Campos, M. P.; Cleveland, G. T.; Plante, I. J.-L.; Owen, J. S., A Tunable Library of Substituted Thiourea Precursors to Metal Sulfide Nanocrystals. Science 2015, 348, 1226-1230. 
(48) Pedetti, S.; Ithurria, S.; Heuclin, H.; Patriarche, G.; Dubertret, B., Type-II CdSe/CdTe Core/Crown Semiconductor Nanoplatelets. J. Am. Chem. Soc. 2014, 136, 16430-16438.

(49) Riedinger, A.; Mule, A. S.; Knusel, P. N.; Ott, F. D.; Rossinelli, A. A.; Norris, D. J., Identifying Reactive Organo-Selenium Precursors in the Synthesis of CdSe Nanoplatelets. Chem. Commun. 2018, 54, 11789-11792.

(50) Hites, R. A.; Biemann, K., Mechanism of Ketonic Decarboxylation. Pyrolysis of Calcium Decanoate. J. Am. Chem. Soc. 1972, 94, 5772-5777.

(51) Harrell, S. M.; McBride, J. R.; Rosenthal, S. J., Synthesis of Ultrasmall and Magic-Sized CdSe Nanocrystals. Chem. Mater. 2013, 25, 1199-1210.

(52) Yu, Q.; Liu, C.-Y., Study of Magic-Size-Cluster Mediated Formation of CdS Nanocrystals: Properties of the Magic-Size Clusters and Mechanism Implication. J. Phys. Chem. C 2009, 113, 12766-12771.
(53) Li, M. J.; Ouyang, J. Y.; Ratcliffe, C. I.; Pietri, L.; Wu, X. H.; Leek, D. M.; Moudrakovski, I.; Lin, Q.; Yang, B.; Yu, K., CdS Magic-Sized Nanocrystals Exhibiting Bright Band Gap Photoemission via Thermodynamically Driven Formation. ACS Nano 2009, 3, 3832-3838.

(54) Tessier, M. D.; Spinicelli, P.; Dupont, D.; Patriarche, G.; Ithurria, S.; Dubertret, B., Efficient Exciton Concentrators Built from Colloidal Core/Crown CdSe/CdS Semiconductor Nanoplatelets. Nano Lett. 2014, 14, 207-213.

(55) Jana, S.; Davidson, P.; Abécassis, B., CdSe Nanoplatelets: Living Polymers. Angew. Chem. Int. Ed. 2016, 55, 9371-9374.

(56) Chen, Y.; Chen, D.; Li, Z.; Peng, X., Symmetry-Breaking for Formation of Rectangular CdSe Two-Dimensional Nanocrystals in ZincBlende Structure. J. Am. Chem. Soc. 2017, 139, 10009-10019. 
Table of Contents Image

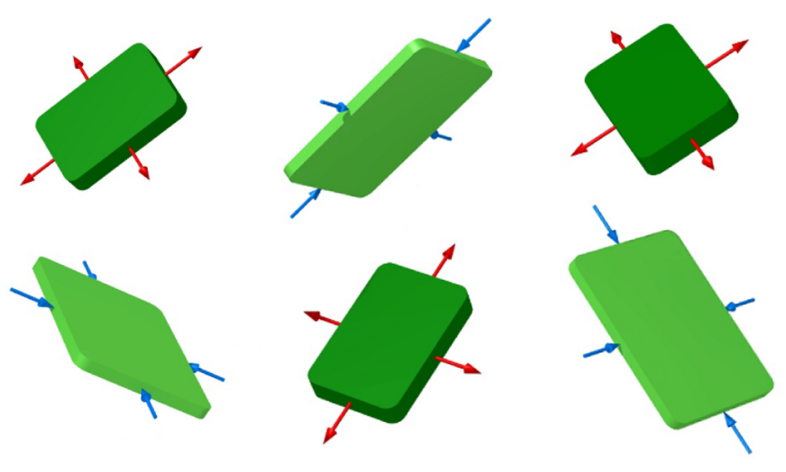




\section{Experimental Evidence for Two-Dimensional Ostwald Ripening in Semiconductor Nanoplatelets}

Philippe N. Knüsel, ${ }^{\dagger}$ Andreas Riedinger, ${ }^{\dagger,}{ }^{\ddagger}$ Aurelio A. Rossinelli, ${ }^{\dagger}$ Florian D. Ott, ${ }^{\dagger}$ Aniket S. Mule, ${ }^{\dagger}$ and David J. Norris, ${ }^{* \dagger}$

${ }^{\dagger}$ Optical Materials Engineering Laboratory, Department of Mechanical and Process Engineering, ETH Zurich, 8092 Zurich, Switzerland

${ }^{\ddagger}$ Max Planck Institute for Polymer Research, Ackermannweg 10, 55128 Mainz, Germany

*e-mail: dnorris@ethz.ch

\section{Supporting Information}

\section{S1. Synthesis of 4-ML-Thick CdS NPLs with Acetyl Sulfide}

We adapted a previously reported synthesis ${ }^{\mathrm{S1}}$ by replacing the elemental sulfur with an equimolar amount of acetyl sulfide. Briefly, $\mathrm{Cd}(\mathrm{OAc})_{2} \cdot 2 \mathrm{H}_{2} \mathrm{O}$ and $\mathrm{OA}$ were dispersed in $\mathrm{ODE}$ and degassed for $1 \mathrm{~h}$ at $110^{\circ} \mathrm{C}$. The flask was subsequently flushed with $\mathrm{N}_{2}$, acetyl sulfide was injected, and the temperature ramped to $240{ }^{\circ} \mathrm{C}$. When the temperature reached $205^{\circ} \mathrm{C}, \mathrm{Cd}(\mathrm{OAc})_{2} \cdot 2 \mathrm{H}_{2} \mathrm{O}$ was added to the reaction mixture. The temperature increase continued to $240^{\circ} \mathrm{C}$, where it was maintained for $15 \mathrm{~min}$. As evidenced by the absorption feature at $406 \mathrm{~nm}$ (Figure S1), the synthesis yielded an almost pure population of 4-ML CdS NPLs. TEM revealed NPLs with lateral dimensions of approximately $10 \times 20 \mathrm{~nm}$, which is comparable to the results obtained with elemental sulfur as the precursor.
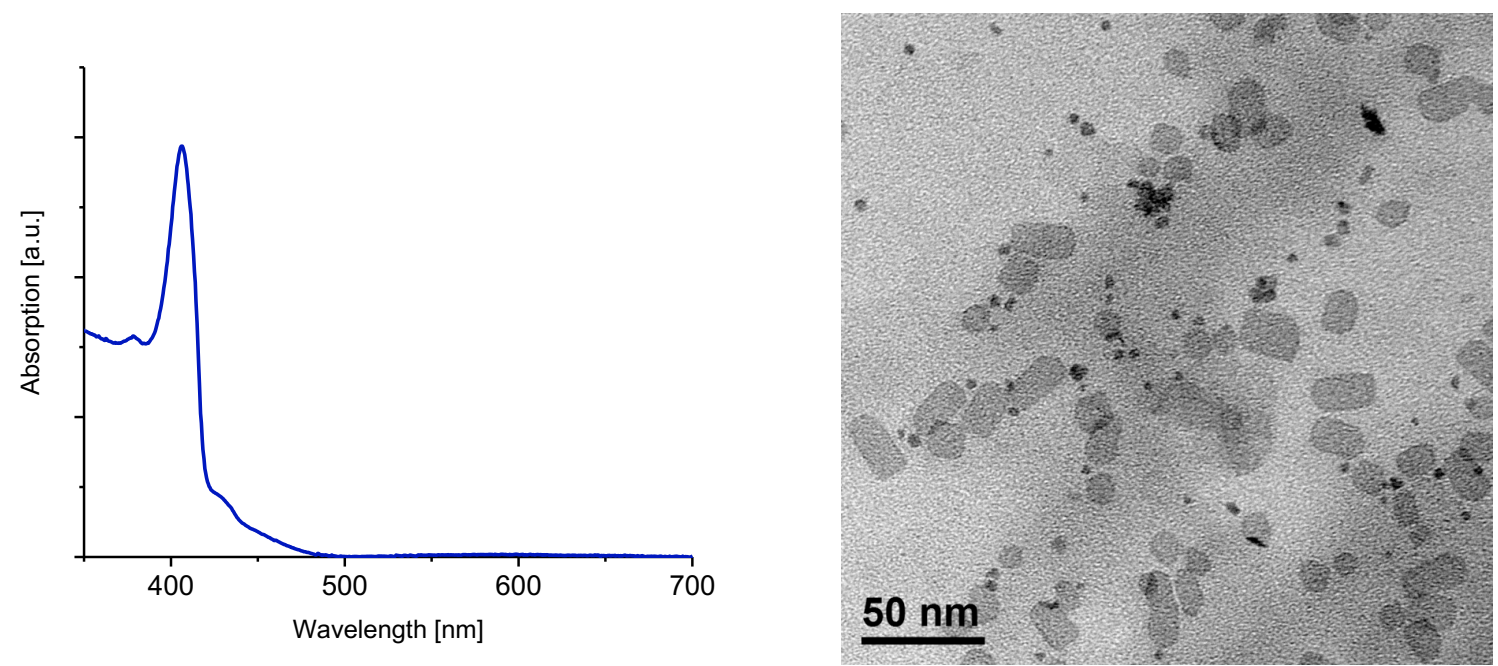

Figure S1. Absorption spectrum (left) and TEM micrograph (right) of 4-ML CdS NPLs obtained with acetyl sulfide as the sulfur precursor. 


\section{S2. Isothermal Colloidal Syntheses of “Baby” CdS NPLs}

In the main text, we describe the use of acetyl sulfide to track the reaction product formed during the temperature ramp in the synthesis of CdS NPLs. Absorption spectroscopy and TEM microscopy (see Figure 1 in the main text) are used to conclude that 2-and 3-ML CdS NPLs are produced that are laterally small (i.e. "baby" CdS NPLs). Here, the same synthetic protocol was adapted to investigate isothermal conditions. After degassing, the reaction mixture was heated to the desired temperature and kept constant. Acetyl sulfide was swiftly injected. Absorption spectra of aliquots taken at different times and temperatures are shown in Figure S2. At temperatures up to $160^{\circ} \mathrm{C}$, the same reaction stages as described in the main text were observed. The temperature only changed the reaction rate. After sufficiently long times (typically one day), NPLs with lateral extensions of more than $10 \mathrm{~nm}$ were observed (Figure S3). At $180^{\circ} \mathrm{C}$, we directly observed the formation of 3- and 4-ML NPLs.

$140^{\circ} \mathrm{C}$

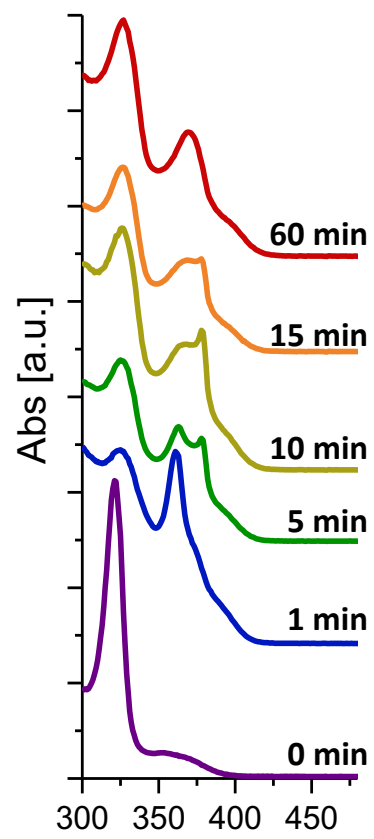

$160^{\circ} \mathrm{C}$

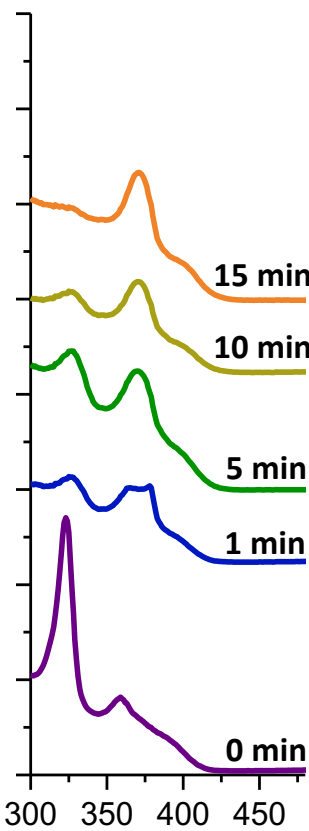

Wavelength [nm] $180^{\circ} \mathrm{C}$

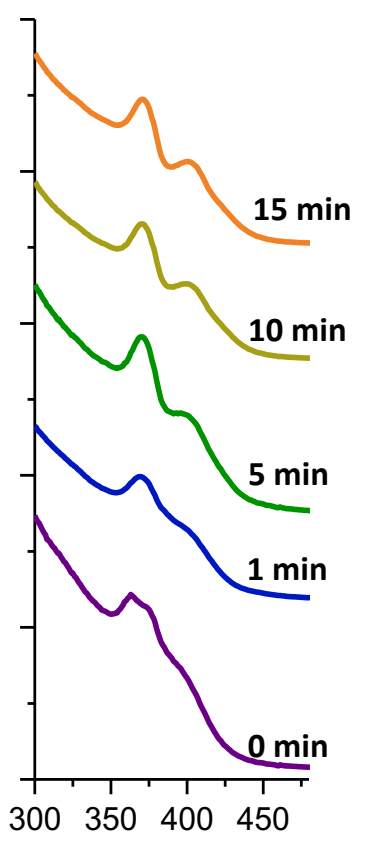

Figure S2. Absorption spectra of aliquots taken at different times for isothermal colloidal syntheses of CdS NPLs using acetyl sulfide as the sulfur precursor.
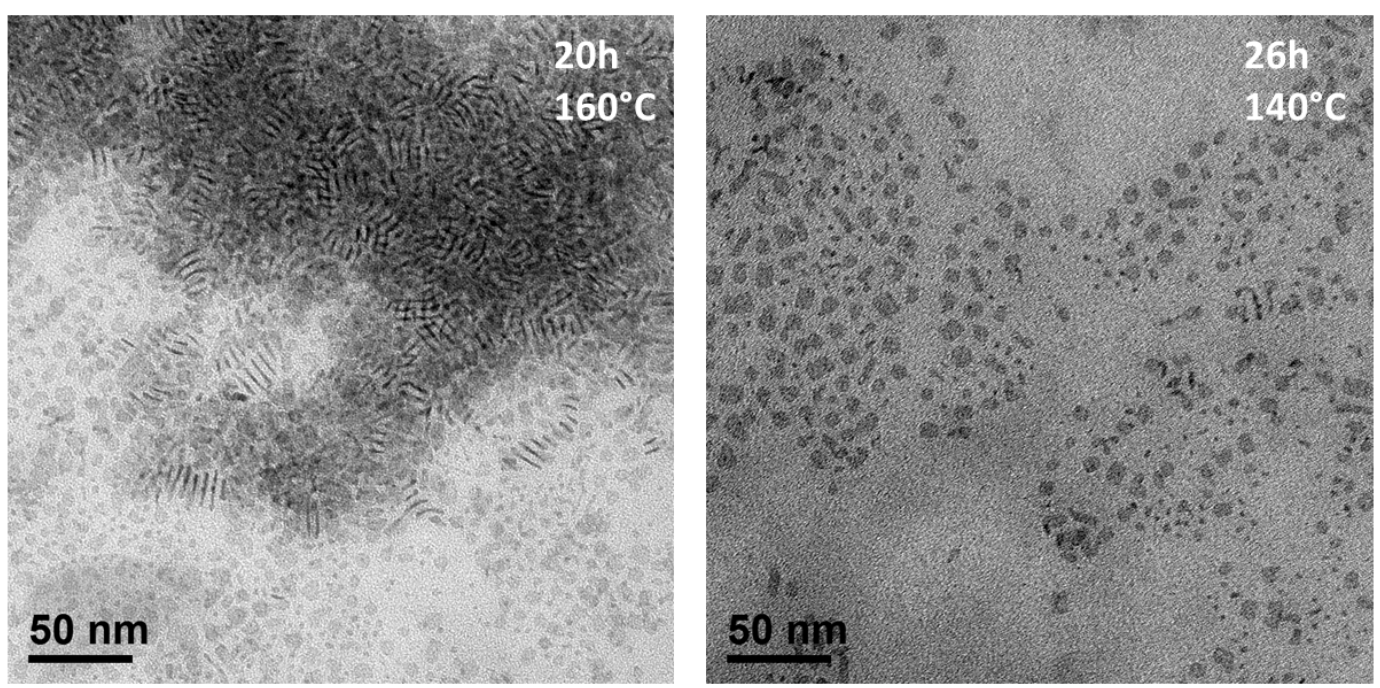

Figure S3. TEM micrographs of CdS NPLs synthesized with acetyl sulfide as the sulfur precursor under isothermal conditions. The growth is maintained at $160^{\circ} \mathrm{C}$ for $20 \mathrm{~h}$ (left) and $140^{\circ} \mathrm{C}$ for $26 \mathrm{~h}$ (right). 


\section{S3. Heating "Baby" CdS NPLs in ODE without $\mathrm{Cd}(\mathrm{OAc})_{2}$}

To confirm the critical role of $\mathrm{Cd}(\mathrm{OAc})_{2}$ in the Ostwald ripening experiments, we conducted a control reaction with the same concentration of "baby" CdS NPLs but without Cd(OAc)2. The "baby" CdS NPLs gradually dissolved, and no thicker NPLs appeared (Figure S4).
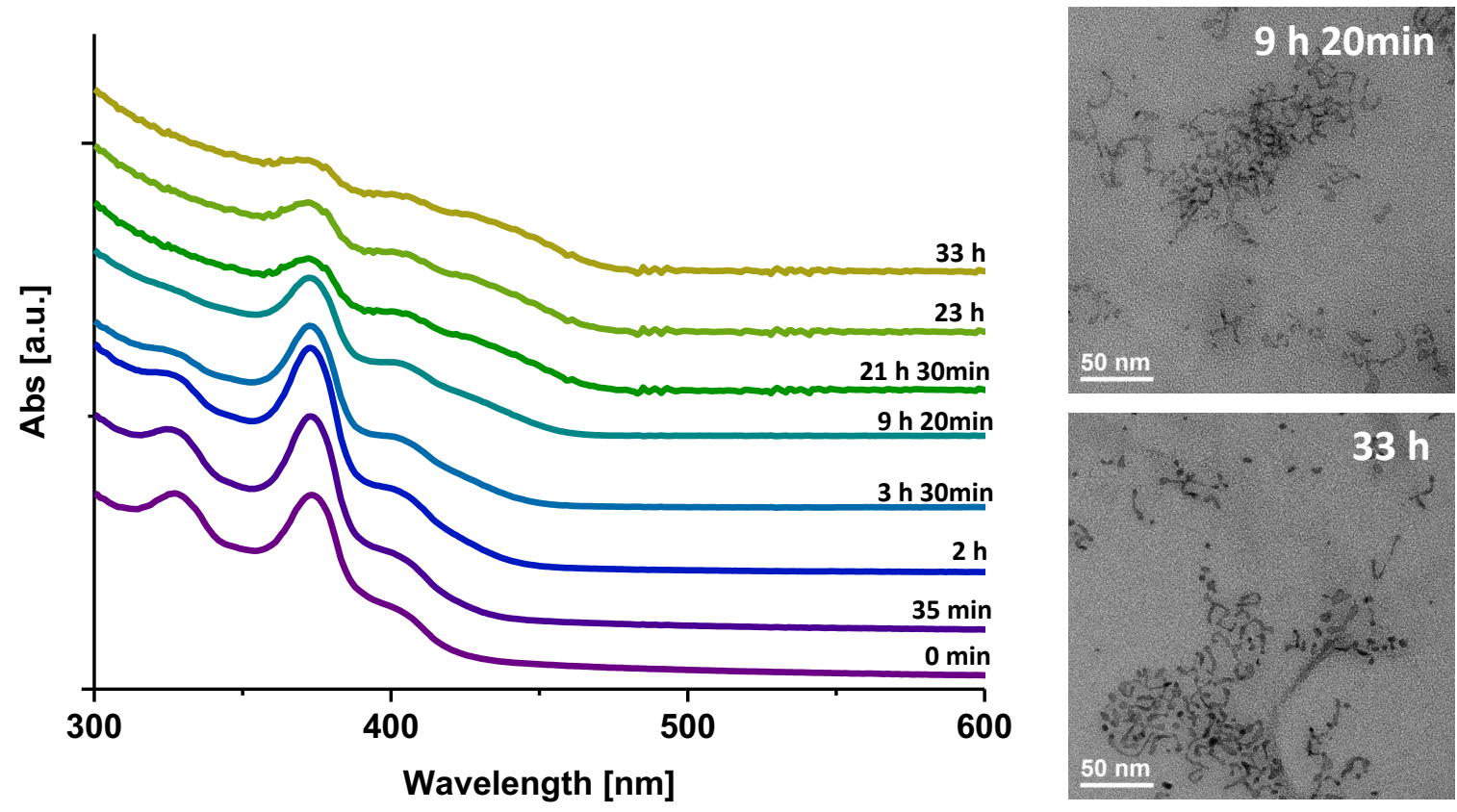

Figure S4. Absorption spectra (left) for aliquots taken from an Ostwald ripening experiment conducted at $180^{\circ} \mathrm{C}$ without added $\mathrm{Cd}(\mathrm{OAc})_{2}$. The absorption peaks gradually disappear as the ripening proceeds with time. TEM micrographs (right) show that nanocrystals with random sizes and shapes are formed.

\section{S4. Ripening Laterally Extended 4-ML CdS NPLs}

We heated laterally extended 4-ML CdS NPLs under similar conditions as the "baby" CdS NPLs. As can be seen from the absorption spectra in Figure S5, no transitions to thicker populations were observed over the experimental time frame (16.5 days).

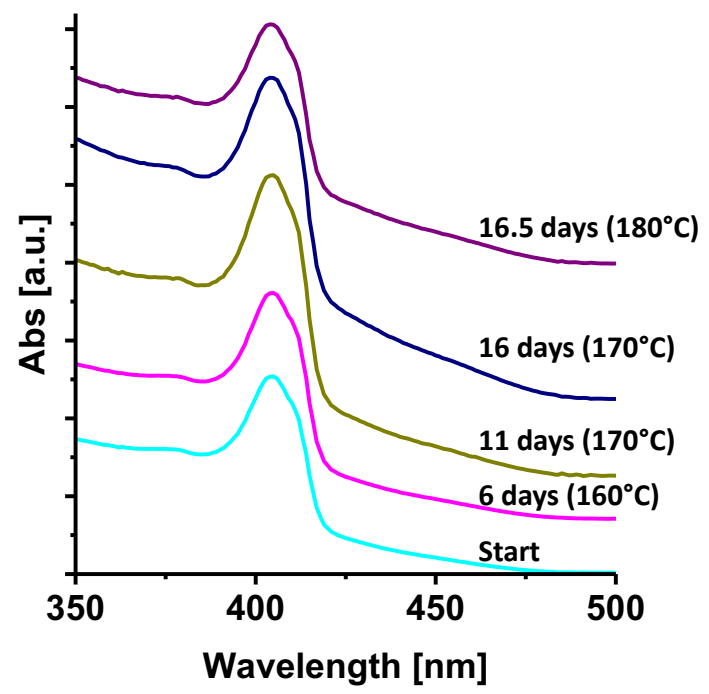

Figure S5. Absorption spectra of laterally extended 4-ML CdS NPLs heated in the presence of $\mathrm{Cd}(\mathrm{OAc})_{2} \cdot 2 \mathrm{H}_{2} \mathrm{O}$ versus time. After 6 days, the temperature was raised to $170^{\circ} \mathrm{C}$, and after 16 days to $180^{\circ} \mathrm{C}$. 


\section{S5. Synthesis of CdSe-CdS Core-Crown NPLs}

When 2- and 3-ML "baby" CdS NPLs were mixed with laterally extended 4-ML CdSe NPLs and heated to $160^{\circ} \mathrm{C}$, we observed a continuous dissolution of the "baby" CdS NPLs. This is seen in the absorption spectra shown in Figure S6. At the same time, absorption peaks for the 4-ML CdSe NPLs remained, and new peaks for 4-ML CdS NPLs appeared. After $20 \mathrm{~h}$, the disappearance of the 2- and 3-ML "baby" CdS NPLs was complete.

Note that the absorption feature at $405 \mathrm{~nm}$ for the 4-ML CdS NPLs is significantly reduced after washing of the mixture with small amounts of ethanol. This is due to selective precipitation of the pure 4-ML CdS NPLs during the purification process.

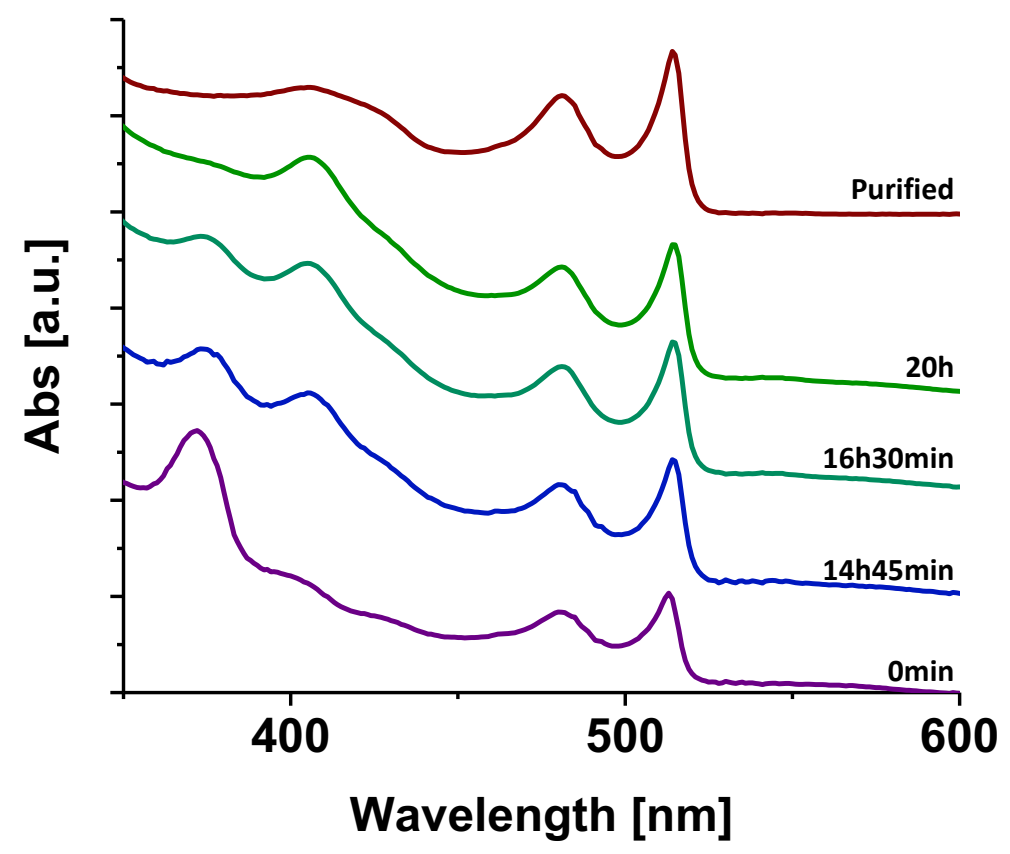

Figure S6. Absorption spectra of aliquots taken during the synthesis of CdSe-CdS core-crown NPLs from laterally extended CdSe NPLs and CdS "baby" NPLs.

However, the photoluminescence excitation (PLE) spectrum (Figure S7), which was taken by monitoring the PL at the CdSe emission peak at $520 \mathrm{~nm}$, shows that an absorption feature at $405 \mathrm{~nm}$ is still present after purification. This indicates that absorption due to 4-ML CdS NPLs is leading to emission from the 4-ML CdSe NPLs. This is consistent with CdSe-CdS corecrown NPLs. In other words, the CdS crown absorbs at $405 \mathrm{~nm}$, the excitation is transferred to the CdSe core, and emission occurs at $520 \mathrm{~nm}$.

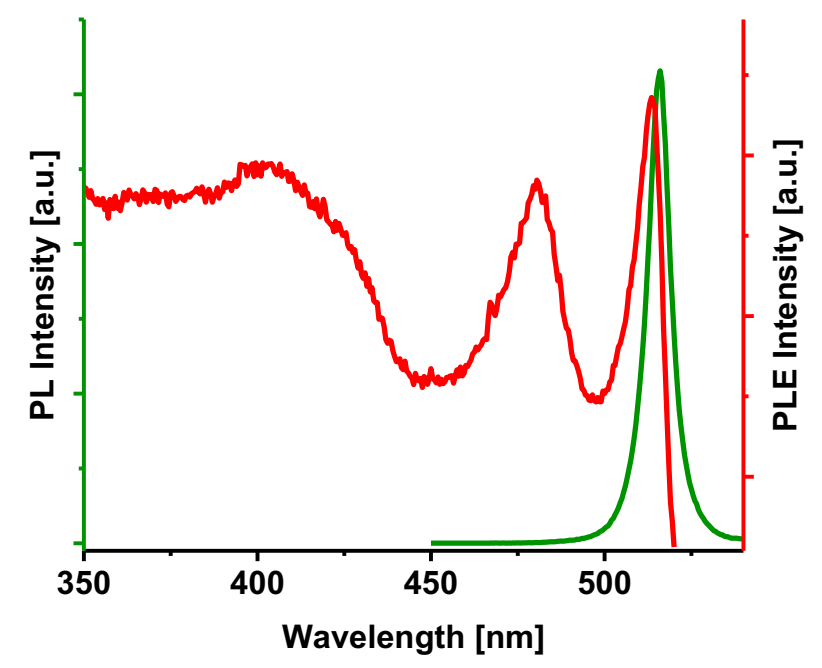

Figure S7. PL (excitation at $400 \mathrm{~nm}$ ) and PLE (emission at $520 \mathrm{~nm}$ ) spectra of CdSe-CdS core-crown NPLs synthesized via ripening. See discussion above and that related to Figure 3 in the main text for further details. 


\section{S6. Supplementary References}

(1) Mahler, B.; Nadal, B.; Bouet, C.; Patriarche, G.; Dubertret, B., Core/Shell Colloidal Semiconductor Nanoplatelets. J. Am. Chem. Soc. 2012, 134, 18591-18598. 